\title{
Protocolisation, utilisation et développement des médicaments anticancéreux dans le cadre de la mise en place de la T2A
}

\author{
Anne Mathieu-Boué, ${ }_{1}$ Pierre Demolis, ${ }^{2}$ Jean-François Bergmann ${ }^{3}$ \\ et les participants à la table Ronde $n^{\circ} 7$ de Giens XXI \\ 1 Novartis Pharma SAS, Rueil Malmaison, France \\ 2 Afssaps, Saint Denis, France \\ 3 Hôpital Lariboisière, Paris, France
}

\begin{abstract}
Mots clés :
bon usage ;

cancérologie ;

protocole thérapeutique ; référentiel;

tarification à l'activité

Résumé - Les médicaments utilisés en cancérologie représentent plus de la moitié des médicaments innovants et coûteux pris en charge hors d'un groupe homogène de soins dans le cadre de la tarification à l'activité. Pour ces médicaments, des référentiels de bon usage élaborés par les sociétés savantes, les agences d'enregistrement et l'Institut National du Cancer (INCa) définissent les indications reconnues, les indications pertinentes et les situations où le traitement ne doit pas être prescrit. Ces référentiels devraient tendre à un bon usage de ces médicaments et permettre un contrôle des prescriptions par les organismes payeurs. Ces référentiels se doivent d'être évolutifs, d'où la nécessité d'une veille bibliographique et de l'appel à des experts indépendants pour une actualisation en fonction des données nouvelles de la science. Les industriels sont impliqués dans cette démarche qui ne doit en rien se substituer aux efforts des développements pouvant conduire à un enregistrement. L'objectif de cette protocolisation est de permettre un accès précoce et légitime de tous les patients aux médicaments représentant un réel progrès thérapeutique. Ces référentiels doivent s'intégrer dans la vie du médicament et doivent permettre de nouveaux développements permettant le bon usage des produits de cancérologie dans des situations correctement validées.
\end{abstract}

\section{Définitions et position du problème}

La mise en place de la tarification à l'activité (dite T2A) implique que, dès janvier 2006, de nouvelles règles s'appliqueront à la facturation par les hôpitaux de certains médicaments signalés comme spécialement innovants ou coûteux. Ces médicaments ne seront pas pris en charge dans le cadre d'un groupe homogène de séjour (médicaments hors GHS) mais feront l'objet d'accords passés entre les établissements hospitaliers et les Agences Régionales de l'Hospitalisation (ARH) dont ils relèvent. Ces accords seront des contrats de bon usage ${ }^{[1]}$ décrivant quelles sont les pratiques dont l'application ne met pas en cause le principe du remboursement. Tout écart à ces contrats devra être dûment justifié, faute de quoi l'hôpital partenaire du contrat s'expose à voir la prise en charge des médicaments hors GHS diminuée ce qui en reporte la

^ Pour la liste des participants, voir en fin d'article. charge sur le budget de l'établissement. Ce dispositif vise donc à encadrer les pratiques et ce ne sont pas les patients qui sont exposés à des sanctions financières mais les hôpitaux au sein desquels les pratiques s'éloignent de l'acceptable.

L'usage d'un médicament dans le cadre de son autorisation de mise sur le marché (AMM) relève évidemment de la pratique validée et reconnue comme telle par les contrats de bon usage. La prescription au cours d'un essai clinique et l'usage dans le cadre d'une autorisation temporaire d'utilisation (ATU) relèvent de réglementations spécifiques auxquelles les contrats de bon usage ne s'appliquent pas. Toute la question de l'écriture des contrats sur cet aspect (ce n'est pas l'unique objet des contrats de bon usage [CBUS] !) réside dans l'acceptation ou le refus de pratiques qui ne correspondent pas à l'AMM.

La liste des médicaments dit «hors GHS » comprend de nombreux anticancéreux. Or, la cancérologie plus que tout autre domaine fait un large usage de pratiques non reconnues de façon 
officielle par les autorités de santé et reposant parfois sur une évidence scientifique solide. Or, la demande d'AMM se fait à l'initiative de l'industriel et certaines situations (indication rares par exemple) peuvent ne pas justifier l'investissement que représente une demande d'enregistrement. D'autre part, il arrive que la demande ait été formulée et que la décision des autorités de régulation soit encore en suspens. Ces pratiques ne sont donc pas toujours reconnues par une AMM.

Pour permettre le respect des engagements pris dans les contrats de bon usage, il importe donc de recenser les pratiques hors $\mathrm{AMM}$ et de les soumettre ensuite à une critique qui permette soit de les considérer temporairement comme admissibles à une prise en charge de même niveau que celle que l'on applique à l'usage de l'AMM soit, au contraire, de les désigner comme non acceptées.

La mise en œuvre des contrats passe donc par la rédaction de référentiels qui prennent en compte les données de la littérature et l'opinion des spécialistes. Pour éviter que des disparités inter-régionales n'apparaissent, il est apparu que ces référentiels devaient être rédigés à un niveau national.

Avant la mise en pratique de la T2A, de nombreuses structures avaient mis en place des groupes de réflexion et élaboré des référentiels applicables à la cancérologie. Ainsi, les Hospices Civils de Lyon, l'Assistance Publique Hôpitaux de Paris, la Fédération Nationale des Centres de Lutte contre le Cancer, les pharmaciens de Centres Hospitaliers Universitaires, l'Observatoire des Médicaments et Innovations Thérapeutiques (OMIT - OMEDIT) Bretagne-Pays de Loire (cette liste n'est pas exhaustive) se sontils déjà dotés de référentiels.

L'écriture des référentiels nationaux se base donc sur ces travaux qu'il s'agit d'harmoniser, de compléter et d'actualiser. L'Institut National du Cancer (INCa) se charge de coordonner le travail d'écriture des référentiels dans le domaine du cancer, en lien avec l'Agence Française de Sécurité Sanitaire et des Produits de Santé (Afssaps) et la Haute Autorité de Santé (HAS). Des réunions préparatoires ont d'ores et déjà fixé la procédure commune d'écriture des référentiels. ${ }^{[2-4]}$

La table ronde organisée à Giens réunissait praticiens, pharmaciens hospitaliers, institutionnels et industriels pour réfléchir aux problèmes pratiques actuellement rencontrés et prévisibles liés à l'élaboration et à l'application des référentiels en cancérologie.

\section{Comment construire un référentiel?}

La conséquence des contrats de bon usage sera binaire : ou bien le médicament utilisé sera admis au remboursement sans pénalité ou bien son usage considéré comme inacceptable exposera l'hôpital à des sanctions financières. Une souplesse est cependant prévue pour des cas individuels exceptionnels, le prescripteur devant alors justifier sa prescription au cas par cas. Cette décision binaire peut être prévue pour toute situation répertoriée et il importe de trier au préalable les pratiques en trois catégories :

- certaines pratiques sont acceptées automatiquement ou sans qu'il soit besoin d'une discussion scientifique extensive : l'AMM ou encore toute situation en passe d'obtenir un avis favorable de l'AMM ;

- d'autres, au contraire sont bien reconnues comme inacceptables et débouchent sur un refus : pratiques ayant fait l'objet d'un refus d'AMM ou signalée comme néfastes par la littérature scientifique;

- la dernière catégorie est celle qui impose la plus grande élaboration lors de l'écriture d'un référentiel : il s'agit des conduites pour lesquelles les données de la littérature sont incomplètes ou discutables sans que l'opinion soit clairement favorable ou défavorable a priori. Lorsque de telles pratiques seront déclarées comme acceptées par le référentiel elles seront décrites dans des «Protocoles Temporaires de Traitement» (PTT). Le PTT est donc un complément provisoire et évolutif à l'AMM. Sa nature temporaire tient au fait que sa validité doit être confirmée à terme par l'octroi d'une AMM ou infirmée soit par le refus de celle-ci soit par l'impossibilité de constituer un dossier propre à l'obtention de l'AMM. Tout PTT est donc assorti d'une date limite de validité et est régulièrement réévalué au regard des nouvelles données scientifiques.

\section{Analyse et réflexions}

Les réflexions concernant les conséquences de l'élaboration des référentiels en cancérologie doivent se faire suivant quatre axes :

- facturation hors GHS : bénéfices attendus et conséquences négatives potentielles;

- évolution d'un référentiel ;

- interaction entre un référentiel établi et la vie d'un médicament ;

- conséquences du dispositif sur la pratique quotidienne du prescripteur.

\section{Facturation hors GHS : bénéfices attendus et conséquences négatives potentielles}

Le premier avantage retenu par les participants de la table ronde a été l'instauration d'une régulation par la qualité (fondée 
sur la revue de la littérature confiée à des experts) là où, jusqu'à présent, la pratique hors AMM n'était pas officiellement reconnue et pouvait ne relever que de décisions isolées éventuellement mal justifiées, d'où des inéquités de traitement entre les patients. L'élaboration de référentiels locaux ou régionaux avant la mise en place de la T2A visait d'ailleurs à instaurer ce contrôle. Encore faut-il que les référentiels soient rédigés clairement en tenant compte des impératifs pratiques liés à l'exercice de la cancérologie.

A cet égard, la rédaction des PTT permet de donner un cadre clair à des pratiques déclarées acceptables à l'issue d'un processus consensuel et scientifiquement justifié.

La publication des référentiels et la mise en place des contrats de bon usage garantira sur tout le territoire une égalité d'accès aux progrès thérapeutiques.

Enfin, le suivi des contrats donnera une information précieuse sur la fréquence des situations relevant de l'application des PTT et comparera rétrospectivement cette fréquence à ce qui était attendu pour valider globalement l'exactitude des situations décrites lors de la demande d'une prise en charge.

En ce qui concerne les inconvénients, outre le risque d'exposer les prescripteurs et les pharmaciens à un surcroît de tâches administratives, les participants ont évoqué le risque de voir les prescriptions se reporter d'un traitement pris en charge dans le cadre des GHS vers un traitement hors GHS, libérant ainsi l'hôpital d'une dépense et imposant à la collectivité nationale une autre dépense, bien plus importante.

\section{Evolution d'un référentiel}

L'écriture des référentiels, commencée en 2005, se poursuivra en 2006 sous l'égide de l'INCa, en lien avec l'Afssaps et la HAS. Des groupes d'experts seront constitués en profitant des groupes déjà fonctionnels présents par exemple au sein des hôpitaux et mettant à profit les documents actuellement disponibles. L'essentiel du travail consistera à décrire ce qui est accepté et à rédiger les décisions correspondantes sous forme de PTT, mais aussi à signaler ce qui n'est pas acceptable. Les PTT peuvent varier en taille, de la simple mention d'une indication (argumentée) jusqu'à un document complet décrivant non seulement l'indication, mais détaillant sa justification, ses limites, les précautions et conditions à respecter ainsi que les mesures mises en place pour aider à la prescription et surveiller les risques associés. Un premier PTT a été publié en Octobre 2005, concernant l'usage de l'Herceptin ${ }^{\circledR}$ dans le traitement adjuvant des cancers du sein surexprimant HER2. La cohérence avec les recommandations de pratique clinique de prise en charge globale de chaque type de cancer sera vérifiée. Les documents seront proposés à la consultation des industriels concernés et seront en fin de processus signés conjointement par les trois autorités de tutelle. La nécessaire actualisation se fera au moins une fois par an en utilisant la littérature et les actes des congrès, les informations fournies par l'industrie pharmaceutique, par les observatoires OMEDIT.

\section{Interaction entre un référentiel établi et la vie d'un médicament}

L'influence de la parution d'un référentiel national sur le développement (international) d'un médicament peut être analysée différemment en tenant compte de deux situations. Si une nouvelle indication faisant l'objet d'un PTT ne fait que précéder l'obtention prévisible d'une AMM et s'intègre à un développement prévu, aucune interaction particulière n'est attendue. En revanche, lorsque la situation reconnue par le référentiel sort du programme de développement prévu par l'industriel, le rôle de celui-ci devient plus complexe.

La transformation d'un PTT en AMM demande un investissement parfois considérable. L'aide de partenaires institutionnels est parfois souhaitable voire indispensable. Dans certains cas, il peut être justifié de réfléchir à une adaptation des niveaux d'exigences (par exemple situation rare où l'essai clinique standard est irréalisable). Dans un premier temps, il peut être raisonnable de renforcer le niveau de preuve sans obligatoirement viser l'obtention d'une AMM.

\section{Conséquences du dispositif sur la pratique quotidienne du prescripteur}

Ce dernier thème de réflexion a impliqué tous les participants et en premier lieu les cancérologues cliniciens. Les référentiels ne seront utiles aux prescripteurs que s'ils sont clairs et concis, décrivant simplement des situations définies par des critères accessibles à la pratique quotidienne.

Les traitements de dernier recours utilisés à titre compassionnel devraient ne pas figurer dans ces référentiels et la consigne générale devrait être de préférer dans ces situations des médicaments moins coûteux et pris en charge dans les GHS.

La prescription d'un traitement hors GHS et en dehors de son AMM devrait relever d'une décision collégiale, soulignant ainsi le passage de pratiques individuelles basées sur une réflexion isolée à des usages décidés en commun en partant de documents de synthèse.

Le respect des référentiels s'articule nécessairement avec la réflexion sur l'auto-évaluation et l'accréditation mais aussi sur les essais cliniques. 


\section{Conclusion}

La mise en place des référentiels en cancérologie est un progrès, et pas seulement du point de vue de la nécessaire maîtrise des dépenses de santé. Les patients pourront bénéficier précocement de progrès validés par une réflexion organisée d'experts, et ce dans le respect de la meilleure égalité possible.

\section{Axes de réflexion et propositions}

Le sujet abordé ici ne débouche pas sur un assortiment de propositions en matière de réflexion ou d'action. En effet, il s'agissait d'observer un processus en développement et de décrire ses conséquences probables sur la prise en charge du cancer en France. Le sujet était donc plus du domaine de la description que de celui de l'investigation ou de la résolution de problèmes. Une nouvelle réflexion sur les bilans de la T2A en cancérologie sera certainement utile dans quelques années et permettra de confronter les vues de 2005 avec celles de 2008 ou 2010.

Dans l'immédiat la mise en place des référentiels dépend d'un accord organisationnel entre l'Afssaps, l'HAS et surtout pour la cancérologie de l'INCa. Il faudra s'assurer de leur validité, de leur évolutivité en fonction des acquisitions de données nouvelles (veille bibliographique), de leur acceptation par tous les prescripteurs, de leur utilisation systématique. Des audits de prescriptions pourraient voir le jour, en particulier par le biais des Commissions du Médicament et du Dispositif Médical Stérile (COMEDIMS) locales. L'analyse de l'usage des référentiels sera faite par les payeurs (ARH [Agence Régionale d'Hospitalisation] et DHOS [Direction de l'Hospitalisation et de l'Organisation des Soins]) selon des modalités qui se doivent d'être mieux définies.

\section{Participants}

Aoustin M. (DGS), Atlani C. (Sanofi Aventis), Bassompierre F. (AP-HP), Benamouzig R. (Hôpital Avicenne, Bobigny), Bonavita M.-J. (AP-HM), Borella L. (INCa), Calvo F. (Hôpital Saint Louis, Paris), Caulin C. (Hôpital Lariboisière, Paris), Cellier D. (Merck Santé), Dahan M. (INCa), Daura V. (AP-HP), De Beels F. (HAS), De Vernejoul N. (HAS), (Diebolt V. (FHF), Dumarcet N. (Afssaps), Durand Zaleski I. (HAS), Fery Lemonnier E. (AP-HP), Genève J. (BECT-FNCLCC), Giri I. (Consultant, Verrières le Buisson), Golinelli D. (DGS), Labreveux C. (Lilly France), Latour J.-F. (Centre Léon Berard, Lyon), Maraninchi D. (Institut Paoli Calmettes, Marseille), Meresse V. (Roche Produits), Mignot L. (Hôpital Foch, Suresnes), Morlet D. (GlaxoSmithKline), Pépin S. (CNAMTS), Ravaud P. (Hôpital Saint André, Bordeaux), Riché C. (CRPV, Brest), Rouleau A. (Afssaps), Tilleul P. (Hôpital Saint Antoine, Paris), Viens P. (Insitut Paoli Calmettes, Marseille).

\section{Références}

1. Décret $n^{\circ} 2005-1023$ du 24 août 2005 relatif au contrat de bon usage des médicaments et des produits et prestations mentionné à l'article L. 162-22-7 du code de la sécurité sociale http://www. legifrance.gouv

2. http://www.e-cancer.fr

3. http://www .afssaps. sante. $\mathrm{fr} / \mathrm{htm} / 3 / \mathrm{t} 2 \mathrm{a} / \mathrm{indt} 2 \mathrm{a} \cdot \mathrm{htm}$

4. http://www anaes. fr

Correspondance et offprints : Jean-François Bergmann, Médecine Interne A, Hôpital Lariboisière, 2 rue Ambroise Paré, 75475 Paris cedex 10, France. E-mail : jf.bergmann@lrb.aphp.fr 